\title{
The post mortem diagnosis of fatal hypoglycaemia using the fatty liver and kidney syndrome of chicks as a model
}

\author{
BY D. W. BANNISTER, IRIS E. O'NEILL AND C. C. WHITEHEAD \\ Agricultural Research Council's Poultry Research Centre, Roslin, Midlothian EH25 9PS, \\ Scotland
}

(Received 7 May 198I-Accepted II November 198I)

\begin{abstract}
1. The concentrations of vitreous humour and plasma glucose were closely correlated in both healthy and fatty liver and kidney syndrome-affected chicks at time of death.

2. The values of vitreous humour glucose and lactate decreased rapidly after death, such that they were not reliable indicators of the presence of hypoglycaemia immediately ante mortem.

3. Hepatic glycogen was extremely low in fatty liver and kidney syndrome-affected birds, whereas significant quantities remained in healthy birds up to at least $24 \mathrm{~h}$ post mortem.
\end{abstract}

Fatty liver and kidney syndrome (FLKS) in young chicks is a condition in which apparently healthy birds, normally 3-5 weeks old, die suddenly, often following minor stress. The condition arises when flocks are given diets marginally deficient in biotin (Payne et al. 1974; Whitehead \& Blair, 1974; Whitehead, Blair et al. 1976). The disorder is quite distinct from classical biotin deficiency which is characterized by poor growth and feathering, perosis and dermatitis, although under certain circumstances both conditions can coexist (Whitehead, Bannister et al. 1976).

Post mortem diagnosis of FLKS is based on characteristic lipid abnormalities, such as fatty infiltration of liver, kidney and other organs (Wight \& Siller, 1975). However, these are secondary changes since the primary metabolic abnormality is failure of hepatic gluconeogenesis via pyruvate carboxylase ( $E C$ 6.4.1.1), a biotin-dependent enzyme (Bannister, 1976). This results in severe hypoglycaemia, which is believed to be the cause of death. (For a review of the metabolic abnormalities associated with FLKS, see Bannister, 1979).

Direct diagnosis post mortem of hypoglycaemia is difficult since histologically-obvious lesions do not occur and plasma concentrations change rapidly after death. The object of the present experiment is to try to identify, using FLKS-induced hypoglycaemia in chicks as a model, post mortem indices that could be of value in determining whether hypoglycaemia is a feature of other conditions and in other species. Of potential interest in the latter context is sudden infant death syndrome (SIDS). Fatty infiltrations of liver and kidneys are not features of SIDS but lower concentrations of hepatic biotin have been reported in SIDS victims than in the livers of infants dying from other known causes (Johnson et al. 1980) and it has been speculated that the primary biochemical lesions in both syndromes may be similar.

\section{MATERIALS AND METHODS}

Procedure

The birds used were broilers obtained 1-d-old from a commercial hatchery (D. B. Marshall Ltd, Newbridge, Scotland). They were housed on floors covered with wood shavings and given either a low-biotin diet similar to one found on previous occasions to cause a high incidence of FLKS (Whitehead \& Blair, 1976) or this diet supplemented with $80 \mu \mathrm{g}$ biotin $/ \mathrm{kg}$. Three categories of bird were used in the experiment. Hypoglycaemic birds were either (a) FLKS-affected chicks which were in the terminal stages of the syndrome (classes 
IV and V of Whitehead (1975)) or (b) dead birds; those which had died from FLKS, usually within a very few minutes and at most not more than $30 \mathrm{~min}$ before experimentation. Controls were healthy birds given the biotin-supplemented diet.

The birds were used when aged 3-5 weeks. Measurements of liver glycogen, vitreous humour glucose and lactate and plasma glucose were made in twenty-five FLKS-affected birds, nine dead birds and fifty controls at time of death. Liver glycogen and vitreous humour glucose and lactate were measured again in groups of five affected or control birds after storage of the carcasses for $1,3,6,18$ or $24 \mathrm{~h}$ in a cold room $\left(2-4^{\circ}\right)$ and also in groups of five controls stored at room temperature $\left(18-21^{\circ}\right)$ for the same periods.

\section{Preparation of samples}

Blood $(1 \mathrm{ml})$ was taken from the wing vein into a heparinized syringe from chicks which were then killed by cervical dislocation. Vitreous humour was collected by removing one eye, puncturing the sclera close to the nerve and applying light pressure. A sample of liver (approximately $2 \mathrm{~g}$ ) was taken from one lobe and cooled immediately with liquid nitrogen. The carcasses were then stored in polyethylene bags at room temperature or in a cold room for appropriate periods of time before further samples were taken from the remainder of the liver and the other eye in a similar manner.

Lactate was measured immediately; glucose and glycogen samples were stored at $-20^{\circ}$ until measurements were made.

\section{Assay methods}

Plasma and vitreous humour glucose were measured in deproteinized samples by a modification of the method of Huggett \& Nixon (1957) in which 4-aminophenazone replaced 0-dianisidine in the assay medium (Trinder, 1969). Values were checked at random by an alternative method employing hexokinase $(E C$ 2.7.1.1) as described by Bergmeyer et al. (1974). Lactate was assayed according to Gutmann \& Wahlefeld (1974) and glycogen by the method of Walaas \& Walaas (1950).

\section{RESULTS}

Table 1 gives the values for the chosen indices at time of death for controls (room temperature and cold-room groups combined), FLKS-affected birds and also for a smaller group of recently-dead birds. As expected, the affected groups exhibited reduced plasma glucose concentrations which were lower than those reported previously (Bannister et al. 1975). In earlier work on FLKS, spuriously-low glucose concentrations were observed on a number of occasions, due to interference with the assay by elevated plasma urate concentrations (Bannister et al. 1974). This possibility was eliminated by random assays on a number of plasma samples using an alternative method (Bergmeyer et al. 1974) which was found to give similar results to those of the routine method (values not shown). Plasma glucose was measured in a number of the recently-dead birds and was significantly lower than in the FLKS-affected group.

Vitreous humour glucose was significantly lower in affected birds than in controls and was further decreased in the dead group although the difference was not significant.

The values for plasma and vitreous humour glucose concentrations of control and FLKS-affected groups were combined and shown to be correlated (Fig. 1). The coefficient of correlation was 0.95 .

Vitreous humour lactate concentration was lower in both affected and dead birds in comparison to controls.

The greatest difference between controls and FLKS-affected chicks was demonstrated by the virtual absence of hepatic glycogen in the latter. The concentration was at the limits of sensitivity of the method used and was less than $1 \%$ of control values. 


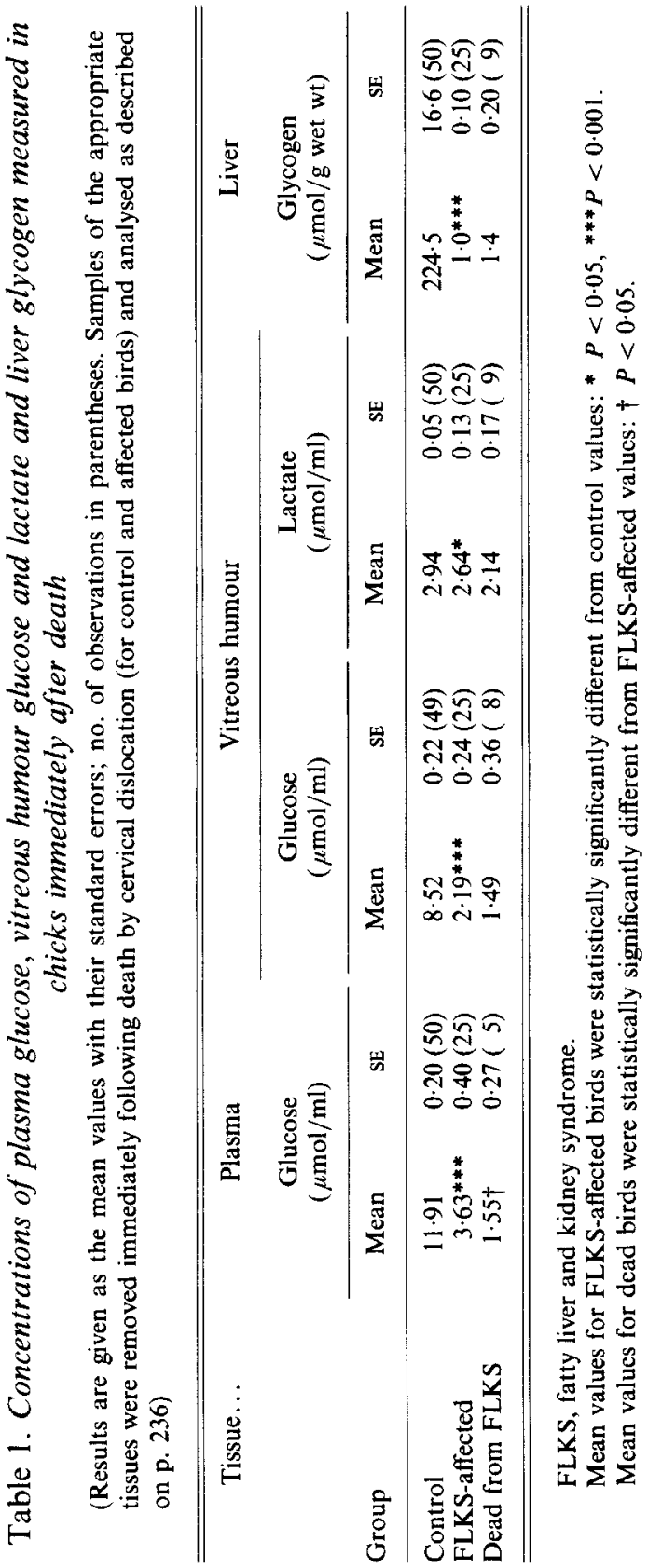




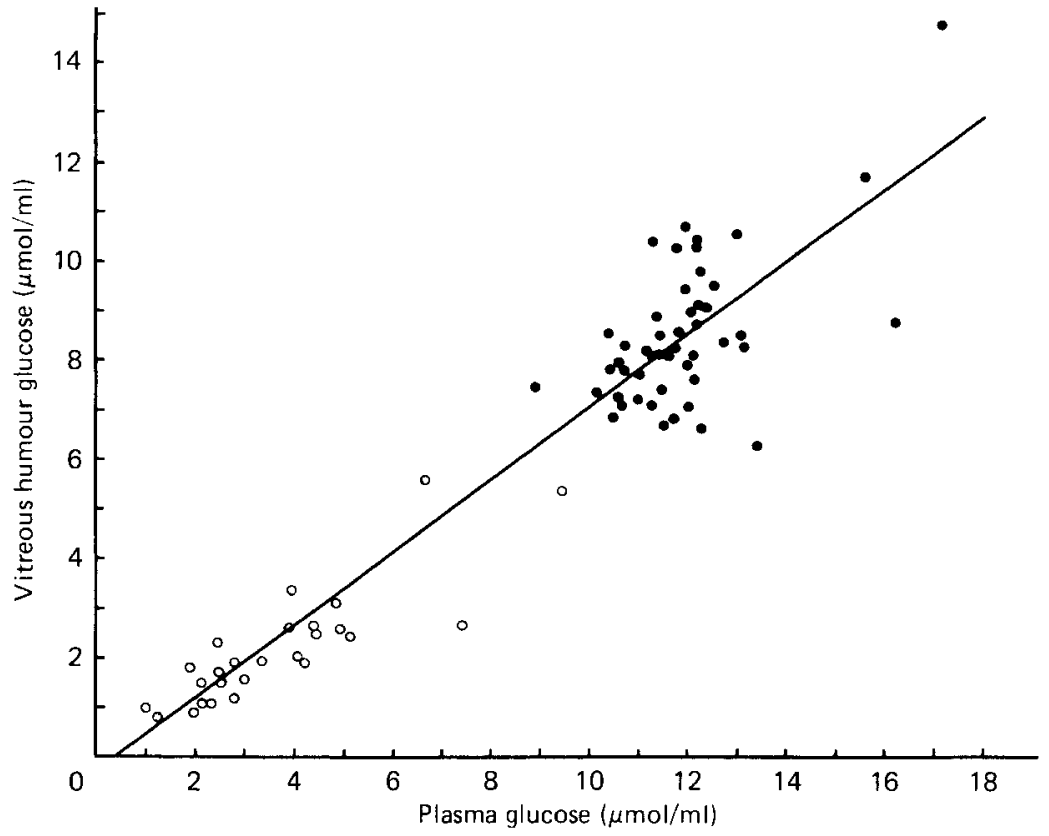

Fig. 1. Relationship between plasma and vitreous humour glucose concentrations $(\mu \mathrm{mol} / \mathrm{ml})$. Glucose concentration was measured in plasma immediately before death and in vitreous humour immediately post mortem in control birds $(\odot)$ and in those affected by fatty liver and kidney syndrome $(O)$.

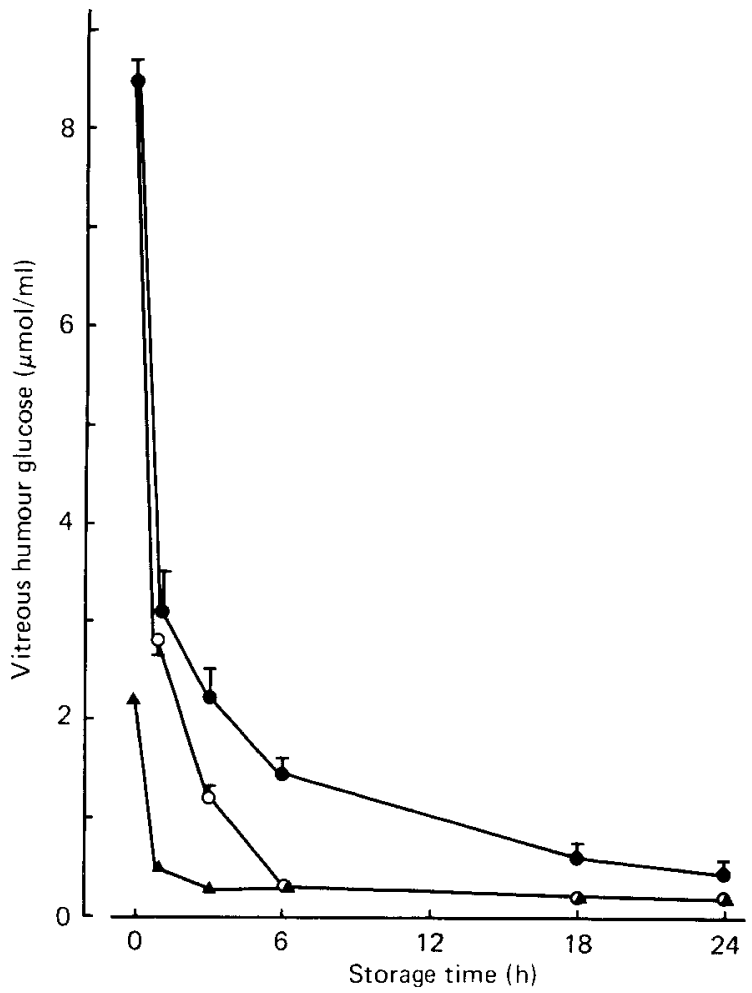

Fig. 2. Loss of vitreous humour glucose on storage. Glucose concentration $(\mu \mathrm{mol} / \mathrm{ml})$ was measured in the vitreous humour of one eye immediately post mortem and subsequently in the other at one of the time intervals indicated. $(\boldsymbol{O})$, Controls, stored at $2-4^{\circ} ;(0)$, controls, stored at room temp. $\left(18-21^{\circ}\right)$; $(\boldsymbol{A})$, fatty liver and kidney syndrome-affected, stored at $2-4^{\circ}$. Points are mean values with their standard errors represented by vertical bars. 


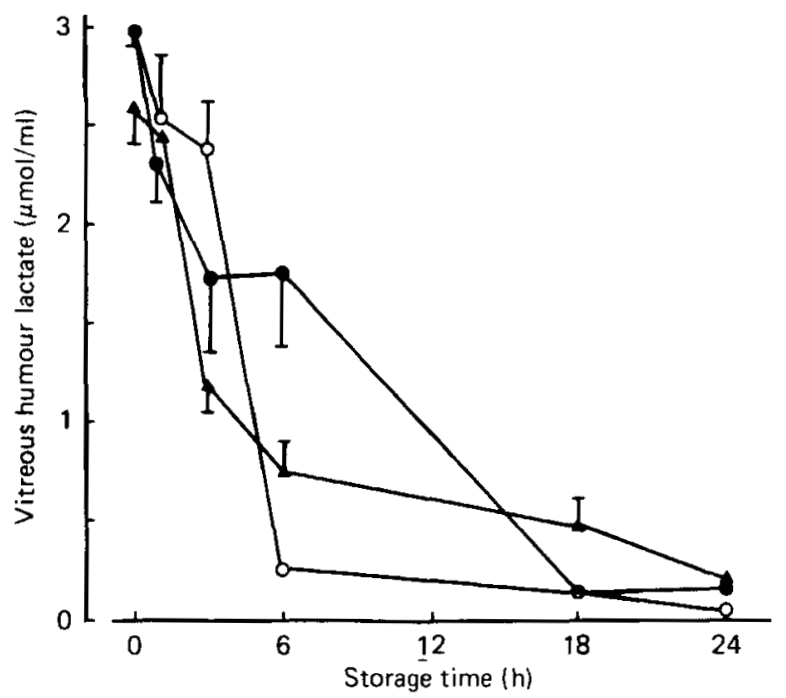

Fig. 3. Loss of vitreous humour lactate on storage. Lactate concentration $(\mu \mathrm{mol} / \mathrm{ml})$ was measured in the vitreous humour of one eye immediately post mortem and subsequently in the other at one of the time intervals indicated. (O), Controls, stored at $2-4^{\circ}$; (O), controls, stored at room temp. $\left(18-21^{\circ}\right)$; (A), fatty liver and kidney syndrome-affected, stored at $2-4^{\circ}$. Points are mean values with their standard errors represented by vertical bars.

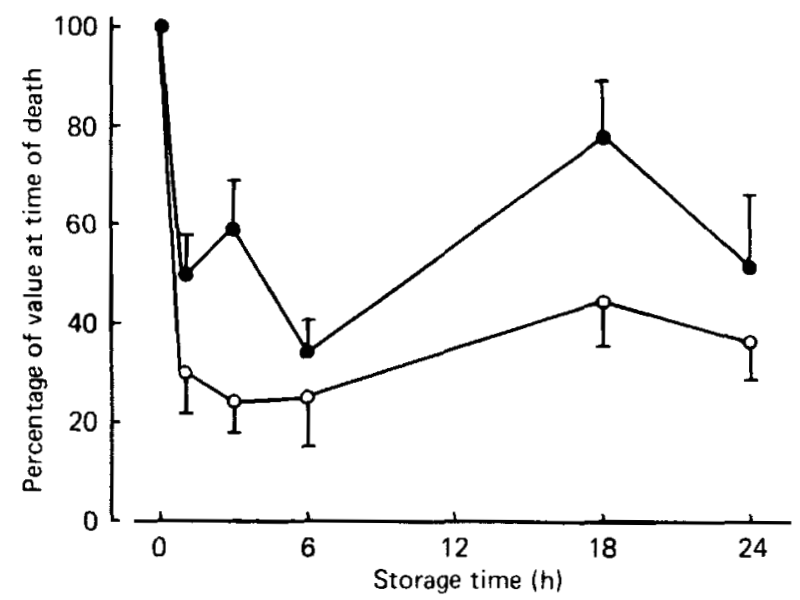

Fig. 4. Loss of liver glycogen on storage. Glycogen concentration ( $\mu$ mol/g wet wt) was measured in a portion of liver removed immediately post mortem and subsequently in another sample taken at one of the time intervals indicated. Due to wide variation in glycogen concentration between birds, values were normalized by expressing them as a percentage of that measured at time of death. (O), Controls, stored at $2-4^{\circ} ;(\bigcirc)$, controls, stored at room temp. $\left(18-21^{\circ}\right)$. Points are mean values with their standard errors represented by vertical bars.

Vitreous humour glucose was lost rapidly following death (Fig. 2), particularly during the first hour post mortem. Storage in the cold room retarded the decline but after $18 \mathrm{~h}$ there was very little glucose remaining. Vitreous humour glucose in affected birds was lower at time of death than in controls (Table 1) and had virtually disappeared $3 \mathrm{~h}$ later (Fig. 2).

Vitreous humour lactate also declined quickly but unevenly after death in all three groups with low values being reached within $18 \mathrm{~h}$ (Fig. 3).

Hepatic glycogen was lost rapidly during the first few hours post mortem from the livers of controls but thereafter remained relatively constant (Fig. 4). The values were normalized 
by presenting them as a percentage of the values measured at the time of death. This was done because of the very large variation in glycogen concentration of the birds used. This phenomenon has been observed previously (Cleland \& Bannister, 1979). Storage at $2-4^{\circ}$ had a beneficial effect on the proportion of glycogen remaining after death. The data on affected birds were not included because glycogen was almost undetectable at time of death.

\section{DISCUSSION}

The hypoglycaemia induced in chicks by FLKS was reflected in changes in the concentrations of the indices examined.

At time of death vitreous humour glucose and plasma glucose concentrations were closely correlated. However, vitreous humour glucose concentration declined rapidly after death such that, in affected birds, it was barely detectable after $6 \mathrm{~h}$ and in controls after $24 \mathrm{~h}$ despite storage at $2-4^{\circ}$.

Vitreous humour lactate concentration was lower in FLKS-affected birds than in controls which is opposite to the finding of elevated plasma lactate in this condition (Hood $e t$ al. 1976; Whitehead et al. 1978). Thus, vitreous humour lactate concentration does not necessarily reflect its status in blood. Since the retina is a glycolytic tissue, it is likely that the reduced concentration in vitreous humour of affected birds reflects a lowered production rate from glucose. The uneven rates of decline in lactate concentrations after death (Fig. 3 ) are probably functions of the rates of loss of the metabolite and rates of formation from glucose.

The above findings suggest that vitreous humour glucose and lactate concentrations are of extremely limited value in post mortem diagnosis of hypoglycaemia in domestic fowl, unless measured very soon after death. Storage at $2-4^{\circ}$ slows down the rate of loss but not sufficiently to extend the usefulness of the measurements appreciably. However, it would be unwise to extrapolate from birds to other species such as man. Avian retina is the most active of glycolytic tissues (Krebs, 1927) and it is possible, therefore, that evidence of hypoglycaemia might persist longer in human vitreous humour due to a slower rate of glycolysis.

During the early part of this investigation, skin glucose concentration was also considered as a possible factor. However, it was also found to decline quickly after death and, because of the relatively tedious methodology involved, it was not pursued further.

The most successful post mortem indicator of hypoglycaemia was hepatic glycogen. Its virtual absence from the livers of affected birds could not be confused with controls, which retained significant glycogen even after $24 \mathrm{~h}$ storage despite wide variation in glycogen concentration between birds. It is likely that any condition causing hypoglycaemia due to failure of gluconeogenesis will be accompanied by depletion of liver glycogen. However, hypoglycaemia due to other causes, for example excessive insulin production, need not be so accompanied.

The observations that sudden infant death sometimes follows minor illness of a nature normally insufficient to cause serious distress, and that the livers of SIDS victims have reduced biotin concentrations (Johnson et al. 1980), suggest that post mortem examination of liver for glycogen should be considered to determine whether hypoglycaemia is a feature of this condition.

The authors are grateful to $\operatorname{Dr}$ A. R. Johnson for suggestions for the work and discussions on the relevance of it to sudden infant death syndrome and to Miss June R. Thomson for her skilled technical assistance. 


\section{REFERENCES}

Bannister, D. W. (1976). Biochem. J. 156, 167.

Bannister, D. W. (1979). Int. J. Biochem. 10, 193.

Bannister, D. W., Burns, A. B., Evans, A. J. \& Martindale, L. (1974). Br. Poult. Sci. 15, 421.

Bannister, D. W., Evans, A. J. \& Whitehead, C. C. (1975). Res. vet. Sci. 18, 149.

Bergmeyer, H. U., Brent, E., Schmidt, F. \& Stork, H. (1974). In Methods of Enzymatic Analysis, vol. 3, p. 1196 [H. U. Bergmeyer, editor]. New York: Academic Press.

Cleland, M. E. \& Bannister, D. W. (1979). Br. Poult. Sci. 20, 307.

Gutmann, I. \& Wahlefeld, A. W. (1974). In Methods of Enzymatic Analysis, vol. 3, p. 1464 [H. U. Bergmeyer, editor]. New York: Academic Press.

Hood, R. L., Johnson, A. R., Fogerty, A. C. \& Pearson, J. A. (1976). Aust. J. Biol. Sci. $29,429$.

Huggett, A. St. G. \& Nixon, D. A. (1957). Biochem. J. 66, 12.

Johnson, A. R., Hood, R. L. \& Emery, J. L. (1980). Nature, New Biol. 285, 159.

Krebs. H. A. (1927). Biochem. Z. 189, 57.

Payne, C. G., Gilchrist, P., Pearson, J. A. \& Hemsley, L. A. (1974). Br. Poult. Sci. 15, 489.

Trinder, P. (1969). Ann. clin. Biochem. 6, 24.

Walaas, O. \& Walaas, E. (1950). J. biol. Chem. 187, 169.

Whitehead, C. C. (1975). Res. vet. Sci. 18, 32.

Whitehead, C. C., Bannister, D. W. \& Cleland, M. E. (1978). Br. J. Nutr. 40, 221.

Whitehead, C. C., Bannister, D. W., Evans, A. J., Siller, W. G. \& Wight, P. A. L. (1976). Br. J. Nutr. $35,115$.

Whitehead, C. C. \& Blair, R. (1974). Wld's Poult. Sci. J. 30, 231.

Whitehead, C. C. \& Blair, R. (1976). Res, vet. Sci. 21, 141.

Whitehead, C. C., Blair, R., Bannister, D. W., Evans, A. J. \& Jones, R. M. (1976). Res. vet. Sci. $20,180$.

Wight, P. A. L. \& Siller, W. G. (1975). Res, vet. Sci. 19, 173. 